\title{
An analysis of IGFBP evolution
}

\author{
Phillip V. Gordon and Marek Marcinkiewicz \\ University of Virginia Children's Hospital, Dept. of Pediatrics, Charlottesville, Virginia
}

\begin{abstract}
Objectives-To perform a synonymous, non-synonymous codon mutational analysis of the IGFBP gene family and identify mechanisms by which the IGFBP subfamilies diverged.

Methods-We identified 78 intact nucleotide sequences from 6 IGFBP subfamilies and 12 different species and used them for phylogenetic and synonymous, non-synonymous codon mutational analysis. Deletion and insertion comparisons were performed across subfamilies to determine if this might play a unique role in subfamily genesis.
\end{abstract}

Results-IGFBP-2 was identified by phylogenetic analysis to be the most related subfamily to the of the IGFBP progenitor, followed by IGFBP-4. Insertions and deletions within the variable domains were association with divergence of each subfamily from its progenitor, suggesting a common motif for IGFBP evolution. Insertions unique to mammals were also found within the amino terminus of IGFBP-2.

Conclusion-IGFBP subfamily divergence is associated with variable domain insertion or deletion and vigorous non-synonymous codon mutation. Our findings suggest strong selective pressure for IGFBP divergence in terrestrial vertebrates.

\section{Keywords}

insulin-like growth factor; IGF binding protein; proteolysis

\section{INTRODUCTION}

Insulin like growth factors -I and -II (IGF-I and IGF-II) have been twin regulators of growth and development throughout vertebrate evolution. However, we understand little in the way of the evolutionary forces that favored the preservation of this duality. At the DNA level, the gene for proto-IGF arose from a duplication of the insulin gene either shortly before or during protochordate evolution around 600 million years ago. ${ }^{1}$ More evolved protochordata (lancets) as well as notochordata (hagfish) also have been found to have a single IGF gene, confirming that proto-IGF maintained its evolutionary niche as a solitary entity for hundreds of millions of years. 2,3 The first documentation of two IGF species within the same animal was found in shark, consistent with a gene duplication event approximately 500 million years ago. ${ }^{4}$

Corresponding author: Phillip V. Gordon MD PhD, PO Box 800386, Dept. of Pediatrics, University of Virginia Children's Hospital, Charlottesville, VA 22908. Telephone:434-924-9971; fax 434-924-2816; E-mail pvg4n@virginia.edu .

Publisher's Disclaimer: This is a PDF file of an unedited manuscript that has been accepted for publication. As a service to our customers we are providing this early version of the manuscript. The manuscript will undergo copyediting, typesetting, and review of the resulting proof before it is published in its final citable form. Please note that during the production process errors may be discovered which could affect the content, and all legal disclaimers that apply to the journal pertain.

Disclosure Statement: the authors have nothing to disclose 
Another important event in IGF evolution was also taking place around the same time as IGF duplication. In boney fish, the dual status of IGFs co-stabilized with the emergence of a half dozen or so distinct IGF binding proteins (IGFBPs). ${ }^{5,6}$ While IGF binding protein activity has been demonstrated as far back in evolution as mollusks ${ }^{7}$ and notochordata ${ }^{8}$ it is, as yet, unproven that the co-emergence of modern IGFBPs with the two IGF species was interdependent. In other words, did this proliferation of constituents arise as a co-regulatory system, in which IGF-I and IGF-II differentially altered the susceptibility of individual IGFBPs to proteolysis? Or, would IGFBPs have arisen anyway, even if only one IGF had been the dominant paradigm in vertebrates? We may never know the answer to this specific question, but more elementary questions may be solvable. For example, did different IGFBPs diverge, at least in part, by responding to selective pressures that have to do with differentially "sensing" the binding of IGF? Such exquisite susceptibility to proteolysis has been well documented, e.g. IGFBP-4 cleavage by pregnancy associated plasma protease A (PAPP-A) and IGFBP-2 by cathepsin $\mathrm{L}$ are each very IGF ligand sensitive. ${ }^{9,10}$

Evolutionary pressure upon the growth hormone (GH)-IGF axis has also been intense for terrestrial vertebrates. Climate change and geographic isolation have forced clades and individual species of from one growth extreme to another, resulting in numerous examples of astonishingly quick shifts in size (both to gigantism and dwarfism; from modern day amphibians, to dinosaurs, birds, and humans from the Isle of Flores). ${ }^{11-15}$ At one point, during the Permian-Triassic extinction, the GH-IGF system may even have been carried forward by a single species of non-amphibian, terrestrial vertebrate (Lystrosaurus - a pre-reptilian creature that may have been the predecessor to both dinosaurs and mammals). ${ }^{16,17}$ Presumably it did so with all six IGFBPs in toto, since these same subfamilies are found in most fish and all mammals.

Indeed, because the IGFBP genes are inversely paired with one another and associated with the HOX gene cluster (of which there is one in amphioxus, two in lamprey, and four in all higher vertebrates - including teleosts) 18,19 , we know with reasonable certainty that all of the duplications necessary to create the full repertoire of IGFBPs originally occurred in boney fish (reviewed by Collet and Candy). ${ }^{20}$ From these four original clusters, we now have left within the mammalian genome two linked and inverted gene pairs: IGFBPs -1 and -3 on chromosome 7, IGFBPs -2 and -5 on chromosome 2, and two unpaired IGFBP genes (IGFBP-4 on chromosome 17 and IGFBP- 6 on chromosome 12). ${ }^{20}$ The current belief is that there was a three step process of IGFBP duplication: IGFBP-2 was duplicated to create IGFBP-5; these were both duplicated to create a second inverted pair (IGFBPs -1 and -3 ); then a third duplication resulted in IGFBPs -4 and -6 . Unfortunately, since these latter genes are neither linked nor paired, one must postulate additional events to satisfy the last step of this theory. One reason the numbers may not add up might be that some of the genes have been lost, or to be more precise, they have devolved into pseudogenes that we have yet to find. This phenomenon is one potential aspect of subfamily divergence. Another aspect of divergence encompasses insertions, deletions and non-synonymous codon mutations that result in divergence of duplicate IGFBP genes into distinct subfamilies. Conversely, some early subfamilies might be highly conserved and remain relatively unchanged throughout vertebrate evolution.

We know that placentation is a crucial point in IGF evolution, since marsupialization has been demonstrated to correlate with a de novo addition to the IGF system (the type II IGF receptor). 21 While little is know about the affect of placentation upon IGFBP mutation, PAPP-A's unique relationship with IGFBP-4 during pregnancy is a good example demonstrating that IGFBP biology did adapt to placentation. ${ }^{21}$ Such changes are likely to be reflected in the genetic record either as new insertions or deletions within IGFBPs specifically involved in placental physiology or as non-synonymous point mutations; small changes in IGFBPs that 
tweak their susceptibility to proteolysis by the new IGFBP proteases associated with pregnancy.

We hypothesized that an analysis of codon mutations in all available IGFBP subfamilies would reveal hot spots of past mutations over the course of vertebrate evolution, potentially providing useful information about the order of IGFBP emergence and refining our understanding of the nature of IGFBP evolution.

\section{METHODS}

\section{Derivation of sequence data and generation of the circular, unrooted tree}

Nucleotide sequence data were retrieved from the public database (PubMed: http://www.pubmed.gov) for species that individually address any aspect of IGFBP phylogeny and manually examined. Nucleotide sequence alignments with alignment conservation, quality, and consensus were assessed with CLUSTAL W, fully automatic program (using all defaults), for global multiple alignment of protein sequences publicly available on the Web (http://www.ebi.ac.uk/). Alignment conservation for all IGFBPs from all available species (that we deemed to be valid) was then transferred as a FASTA file into Biology Workbench (http://workbench.sdsc.edu/) to transform the FASTA file into a Newick file. This file was then uploaded into Tree Explorer using the PHYLIP package with neighbor joining (NJ) and a circular, unrooted phylogenetic tree was generated. It was not possible to generate an out group (as is often done with a single gene across multiple phyla comparisons), since only one nonvertebrate IGFBP-like protein has ever been sequenced (in mollusks). ${ }^{7} \mathrm{We}$ also did not include any mammalian IGFBP-related proteins in our analysis as the use of six closely related subfamilies improves the validity of our phylogenetic analysis, whereas the inclusion of more poorly related relatives would reduce the validity of an unrooted tree.

\section{Synonymous, non-synonymous analysis of IGFBP mutations}

To analyze insertion / deletion, synonymous, and non-synonymous IGFBP mutations, we accessed the SNAP: Synonymous Non-synonymous Analysis Program on line at the following link: (http://www.hiv.lanl.gov/content/hiv-db/SNAP/WEBSNAP/SNAP.html). We then uploaded the aligned nucleotide FASTA file into SNAP and calculated the cumulative codon behavior. This data was then exported to Excel and plotted, first individually as each separate form of mutation and then as an integral in which insertion / deletions were added with nonsynonymous deletion and then synonymous deletions were subtracted from this sum integral. This plot was then overlaid with its own linear regression (using Excel) to demonstrate hot spots of conservation versus evolutionary selection pressure.

\section{RESULTS}

\section{Phylogenetic analysis}

We found 78 suitable, full length nucleotide IGFBP sequence using PubMED (See supplement 1 for PubMED accession numbers). These sequences were aligned using CLUSTAL W. The resulting FASTA file was loaded into Biology Workbench (http://workbench.sdsc.edu/), then transformed into a Newick file to facilitate the building of an unrooted, circular phylogenetic tree (Figure 1). This topology has the advantage of combining both radial branching and accurate evolutionary distance within a single plot. Thus we were able to observe the IGFBP subfamily which most closely approximates the IGFBP progenitor as the one with the shortest internal branches at the center of the tree. In this case, IGFBP-2 was the closest relative to the center, with IGFBP-4 being the next of kin. We note that IGFBP-4 was the only subfamily with no fish species represented within it, however, rainbow trout partial length fish IGFBP-4 has been identified and cloned - so IGFBP-4 did originate within aquatic vertebrates. 22 
IGFBPs 1, 3 and 5 had similar evolutionary distances from the origin and near equidistant branching, suggesting that the current versions may have diverged at similar times. We found one interesting caveat in that kissing fish and salmon had examples of "IGFBP-3" off of a distal IGFBP-2 branch when compared to all other vertebrate IGFBP-3s (which share a common branch with IGFBP-5), raising the conundrum of a seventh IGFBP subfamily. Likewise, a single example of an "IGFBP-1" in salmon was found to have branched off of the progenitor branch for the IGFBP-6 subfamily, raising the possibility of a eighth IGFBP subfamily.

\section{Synonymous, non-synonymous analysis of IGFBP mutations}

Analysis of synonymous and non-synonymous mutations revealed that non-synonymous mutations were cumulatively the most abundant, followed by insertions / deletions and then synonymous mutations (Figure 2). A look at specific regions of mutation revealed that two cysteine-rich domains were highly conserved (codons 84-141 and 286-331, using our multialignment system) with regard to insertions and deletions, but that non-synonymous mutations occurred throughout the gene, with the exception of the distal carboxyl terminus (codons 352394 - potentially suggesting an important transcriptional role for that region throughout the family). Synonymous mutations were less common and occurred in exactly the same distribution as non-synonymous mutations, indicating that there were no hot spots of selective point mutation.

\section{Summary integral of synonymous / non-synonymous analysis}

A summary integral consisting of the addition of the insertion / deletion mutations with the non-synonymous mutation, minus the synonymous mutation, resulted in a plot of peaks and valleys that illustrated hot spots of selective pressure and conservation respectively (Figure 3 A). These areas were then bisected from one another by overlaying the plot with a linear regression (yielding a y intercept $=15.07$ and a slope $=-0.15$ ). We found two hot spots of selection pressure within the amino terminus and three within the variable domain. Upon inspection of the IGFBP amino acid multi-sequence alignment, there were representative insertions or deletions within all six IGFBPs that corresponded with these hotspots, suggesting that insertion / deletion was a crucial mechanism of divergence for the IGFBP subfamilies (Figure 3 B).

\section{Analysis of Insertion and Deletion "Hot Spots"}

IGFBPs $1,3,4,5$ and 6 have each had insertions or deletions within the variable domains that correspond with the codon hot spots for increased mutation rates. However, the variable domain of IGFBP-2 has been extremely stable with regard to insertions and deletions in both fish and mammals, suggesting that this specific subfamily domain has been conserved intact throughout the history of IGFB evolution. In combination with our unrooted tree, this provides additional evidence that IGFBP-2 is the common progenitor for all other IGFBPs.

The overlapping insertions found within the variable domains of IGFBPs -3 and -5 (codons 163-175 and 158-180) suggest that these IGFBPs might have had common origins, either via duplication events and subsequent selective pressure for deletion and mutation of individual codons or simply by selective pressure for similar insertion events. Likewise, similar overlapping deletion events are evident in IGFBPs -1 and -4 and the same postulates can be made for this pair of IGFBPs. IGFBP-6 stands out as having a unique domain of variable domain deletion. When comparing this to our unrooted tree, one finds that insertions and deletions are predictive of the branching distances between each IGFBP subfamily, with IGFBP-4 and -1 being the closest to IGFBP-2 and IGFBP- 6 being the most distant.

IGFBP-2 has also had relatively recent insertions in the amino terminus (two in rodents and one in all other mammals and chicken) which was extremely interesting, since IGFBP-2 is 
abundant during parturition. The chicken insertion (codons 22-31) contains three arginines not found in any of the corresponding mammalian insertions, suggesting that the loss or divergence of these three codons may be an important distinction between therians and avians. Likewise, the additional amino terminus insertions in rodents (codons 77-91) is of extreme interest and suggests an even more recent evolutionary adaptation within IGFBP-2 that is unique to rodents.

Finally we found that the distal amino terminus and proximal carboxyl terminus were highly conserved regions. This was anticipated since these encompassed the cysteine rich domains that give IGFBPs their common tertiary structure

\section{DISCUSSION}

Use of the un-rooted, circular tree topology suggests that IGFBP-2 is closest to the progenitor IGFBP. Based on this technique, IGFBP-4 is the next oldest modern relative to have emerged with IGFBP-1 being a distant third followed very closely by IGFBPs -3 and -5 with IGFBP- 6 being the most recent subfamily to diverge. Specific comparisons of subfamily insertions and deletions within the variable domains correlate very well with this ordering, suggesting that insertions and deletions have played an important role in the divergent maturation of the six subfamilies. We stress that the timing of divergence and the timing of the origins for IGFBP subfamilies are not synonymous and this work in no way challenges the three step duplication theory of IGFBP subfamily origin. ${ }^{20}$

It is well documented that duplication events surrounding the HOX sites in notochordata and boney fish are responsible for the formation of the IGFBP subfamilies. These linkage studies suggest that at one time as many as eight IGFBPs must have existed (two for each four HOX sites). ${ }^{19}$ Our unrooted tree supports the eight IGFBP subfamily postulate, as there is a seventh "IGFBP-3" subfamily in salmon and kissing fish that is unrelated to the traditional IGFBP-3 subfamily and an eighth "IGFBP-1" subfamily in salmon that is poorly related to the IGFBP-1 subfamily. Thus it remains entirely possible that eight IGFBP subfamilies once existed in boney fish but only six functional niches for IGFBPs remain in terrestrial vertebrates.

The value of codon mutational analysis is to assess the selection pressures that conserved or forced divergence of each distinct IGFBP subfamily. To that end, our analysis suggests that variable domain insertions / deletions represent a substantial mechanism for change within IGFBPs. Non-synonymous codon mutations occurred in a more dispersed distribution (although there were few codon mutations, either synonymous or non-synonymous in the distal carboxyl terminus) and were collectively more numerous. Together, these two forces represent tremendous selective pressure for IGFBP divergence (based on comparison to the lower basal rate of synonymous codon mutations).

Our findings also suggest novel evolutionary relationships between IGFBPs. IGFBPs -3 and -5 each share a common variable domain insertion site. Likewise, IGFBPs -1 and -4 share overlapping deletion sites. These findings could be consistent with each IGFBP pair being part of a duplication event (an explanation that would go against the three step, HOX linkage theory) or, more likely, that each of these IGFBP pairs experienced similar selection pressures, favoring progeny who acquired similar insertions / deletions. This might explain why the alternate subfamilies of "IGFBP-3" and "IGFBP-1" in salmon and kissing fish may have become lost genes in higher vertebrates, (because salmon and kissing fish "IGFBP-3" lack the IGFBP-3 variable domain insert and salmon "IGFBP-1" has an atypical IGFBP-1 variable domain deletion). Additional sampling will be required to test these competing hypotheses.

If there is such tremendous evolutionary pressure upon individual IGFBPs to diverge, the obvious question must be "why?". What could possibly exert such strong selective pressure? The most likely case would be a change in susceptibility to existing IGFBP proteases. 
Experiments with IGFBP-2 variable domain deletion mutants have revealed dramatic alterations in IGFBP protease susceptibility and a concomitant loss of protease sensitivity to IGF binding (unfortunately, we are unable to publish these compelling results without risking patent infringement). These findings suggest that insertions and deletions within the variable domain could profoundly affect protease specificity and IGF ligand responsiveness. Such an effect would potentially result in an immediate shift in the IGF axis within an organism where IGFBPs lack redundancy. Dr Cunming Duan has repeatedly demonstrated this phenomenon in zebrafish, where mutations in IGFBPs 1, 2 and 3 result in profoundly perturbed phenotypes in tissues that express a single IGFBP subfamily. ${ }^{23-25}$ In contrast, mammals tend to express multiple IGFBPs in every tissue, and thus Dr John Pintar's six strains of IGFBP knock out mice demonstrate relatively normal phenotypes. ${ }^{26-28}$ This might explain why six IGFBPs is the magic number. Six may be the number beyond which all additional IGFBP variation is truly redundant and thus there is no selective pressure for conservation of the additional IGFBP subfamilies.

\section{Summary}

IGFBPs have diverged into six subfamilies through evolutionary mechanisms that include gene duplication in boney fish, ${ }^{19}$ punctuated insertion / deletion within amino and variable domain hot spots and continuous refinement throughout most of the gene by point mutation. Two exceptions are the highly conserved cysteine rich areas in the distal amino and the proximal carboxyl domains, whereas most other regions have proven mutable and therefore favorable to adaptation. Finally, insertions or deletions within the variable domain likely represent the critical step towards functional divergence from a duplicated progenitor IGFBP gene into a unique IGFBP subfamily.

\section{Acknowledgments}

Dr Gordon received support from NIH Grant 1KO8DK/HD61553-01.

\section{References}

1. McRory JE, Sherwood NM. Ancient divergence of insulin and insulin-like growth factor. DNA Cell Biol 1997;16:939-949. [PubMed: 9303436]

2. Chan SJ, Cao QP, Steiner DF. Evolution of the insulin superfamily: cloning of a hybrid insulin/insulinlike growth factor cDNA from amphioxus. Proc Natl Acad Sci U S A 1990;87:9319-9323. [PubMed: 1701257]

3. Nagamatsu S, Chan SJ, Falkmer S, Steiner DF. Evolution of the insulin gene superfamily. Sequence of a preproinsulin-like growth factor cDNA from the Atlantic hagfish. J Biol Chem 1991;266:23972402. [PubMed: 1989990]

4. Duguay SJ, Chan SJ, Mommsen TP, Steiner DF. Divergence of insulin-like growth factors I and II in the elasmobranch, Squalus acanthias. FEBS Lett 1995;371:69-72. [PubMed: 7545136]

5. Hwa V, Oh Y, Rosenfeld RG. The insulin-like growth factor-binding protein (IGFBP) superfamily. Endocr Rev 1999;20:761-787. [PubMed: 10605625]

6. Kamangar BB, Gabillard JC, Bobe J. Insulin-like growth factor-binding protein (IGFBP)- 1, -2, -3, -4, -5 , and -6 and IGFBP-related protein 1 during rainbow trout postvitellogenesis and oocyte maturation: molecular characterization, expression profiles, and hormonal regulation. Endocrinology 2006;147:2399-2410. [PubMed: 16439447]

7. Weiss IM, Gohring W, Fritz M, Mann K. Perlustrin, a Haliotis laevigata (abalone) nacre protein, is homologous to the insulin-like growth factor binding protein $\mathrm{N}$-terminal module of vertebrates. Biochem Biophys Res Commun 2001;285:244-249. [PubMed: 11444832]

8. Upton Z, Chan SJ, Steiner DF, Wallace JC, Ballard FJ. Evolution of insulin-like growth factor binding proteins. Growth Regul 1993;3:29-32. [PubMed: 7683521] 
9. Fowlkes J, Freemark M. Evidence for a novel insulin-like growth factor (IGF)-dependent protease regulating IGF-binding protein-4 in dermal fibroblasts. Endocrinology 1992;131:2071-2076. [PubMed: 1385096]

10. Gordon PV, Paxton JB, Kuemmerle JF, Fox NS. A 14-kDa cathepsin L-derived carboxyl IGFBP-2 fragment is sequestered by cultured rat ileal crypt cells. Am. J. Physiol. Gastrointest Liver Physiol 2005;289:G79-G87. [PubMed: 15705658]

11. Hershkovitz I, Kornreich L, Laron Z. Comparative skeletal features between Homo floresiensis and patients with primary growth hormone insensitivity (Laron Syndrome). Am J Phys Anthropol 2007;134:198-208. [PubMed: 17596857]

12. Turner AH, Pol D, Clarke JA, Erickson GM, Norell MA. A basal dromaeosaurid and size evolution preceding avian flight. Science 2007;317:1378-1381. [PubMed: 17823350]

13. Tryjanowski P, Sparks T, Rybacki M, Berger L. Is body size of the water frog Rana esculenta complex responding to climate change? Naturwissenschaften 2006;93:110-113. [PubMed: 16514516]

14. Bunce M, Szulkin M, Lerner HR, Barnes I, Shapiro B, Cooper A, Holdaway RN. Ancient DNA provides new insights into the evolutionary history of New Zealand's extinct giant eagle. PLoS Biol 2005;3:e9. [PubMed: 15660162]

15. Baker AJ, Huynen LJ, Haddrath O, Millar CD, Lambert DM. Reconstructing the tempo and mode of evolution in an extinct clade of birds with ancient DNA: the giant moas of New Zealand. Proc Natl Acad Sci U S A 2005;102:8257-8262. [PubMed: 15928096]

16. Olsen PE, Shubin NH, Anders MH. New early Jurassic tetrapod assemblages constrain TriassicJurassic tetrapod extinction event. Science 1987;237:1025-1029. [PubMed: 3616622]

17. Modesto S, Sues HD, Damiani R. A new Triassic procolophonoid reptile and its implications for procolophonoid survivorship during the Permo-Triassic extinction event. Proc Biol Sci 2001;268:2047-2052. [PubMed: 11571052]

18. Holland PWH, Garcia-Fernandez J, Williams NA, Sidow A. Gene duplications and the origins of vertebrate development. Development 1994:125-133.

19. Bailey WJ, Kim J, Wagner GP, Ruddle FH. Phylogenetic reconstruction of vertebrate Hox cluster duplications. Mol. Biol. Evol 1997;14:843-853. [PubMed: 9254922]

20. Collet C, Candy J. How many insulin-like growth factor binding proteins? Mol Cell Endocrinol 1998;139:1-6. [PubMed: 9705068]

21. Killian JK, Buckley TR, Stewart N, Munday BL, Jirtle RL. Marsupials and Eutherians reunited: genetic evidence for the Theria hypothesis of mammalian evolution. Mamm Genome 2001;12:513517. [PubMed: 11420613]

22. Kamangar BB, Gabillard JC, Bobe J. Insulin-like growth factor-binding protein (IGFBP)-1, -2, -3, $-4,-5$, and -6 and IGFBP-related protein 1 during rainbow trout postvitellogenesis and oocyte maturation: molecular characterization, expression profiles, and hormonal regulation. Endocrinology 2006;147:2399-2410. [PubMed: 16439447]

23. Kajimura S, Aida K, Duan C. Insulin-like growth factor-binding protein-1 (IGFBP-1) mediates hypoxia-induced embryonic growth and developmental retardation. Proc Natl Acad Sci U S A 2005;102:1240-1245. [PubMed: 15644436]

24. Wood AW, Schlueter PJ, Duan C. Targeted knockdown of insulin-like growth factor binding protein-2 disrupts cardiovascular development in zebrafish embryos. Mol Endocrinol 2005;19:1024-1034. [PubMed: 15618288]

25. Li Y, Xiang J, Duan C. Insulin-like growth factor-binding protein-3 plays an important role in regulating pharyngeal skeleton and inner ear formation and differentiation. J Biol Chem 2005;280:3613-3620. [PubMed: 15550380]

26. Ning Y, Schuller AG, Bradshaw S, Rotwein P, Ludwig T, Frystyk J, Pintar JE. Diminished growth and enhanced glucose metabolism in triple knockout mice containing mutations of insulin-like growth factor binding protein-3, -4, and -5. Mol Endocrinol 2006;20:2173-2186. [PubMed: 16675541]

27. van Kleffens M, Groffen CA, Dits NF, Lindenbergh-Kortleve DJ, Schuller AG, Bradshaw SL, Pintar JE, Zwarthoff EC, Drop SL, van Neck JW. Generation of antisera to mouse insulin-like growth factor binding proteins (IGFBP)-1 to -6: comparison of IGFBP protein and messenger ribonucleic acid localization in the mouse embryo. Endocrinology 1999;140:5944-5952. [PubMed: 10579362] 
28. Pintar JE, Schuller A, Cerro JA, Czick M, Grewal A, Green B. Genetic ablation of IGFBP-2 suggests functional redundancy in the IGFBP family. Prog Growth Factor Res 1995;6:437-445. [PubMed: 8817688] 


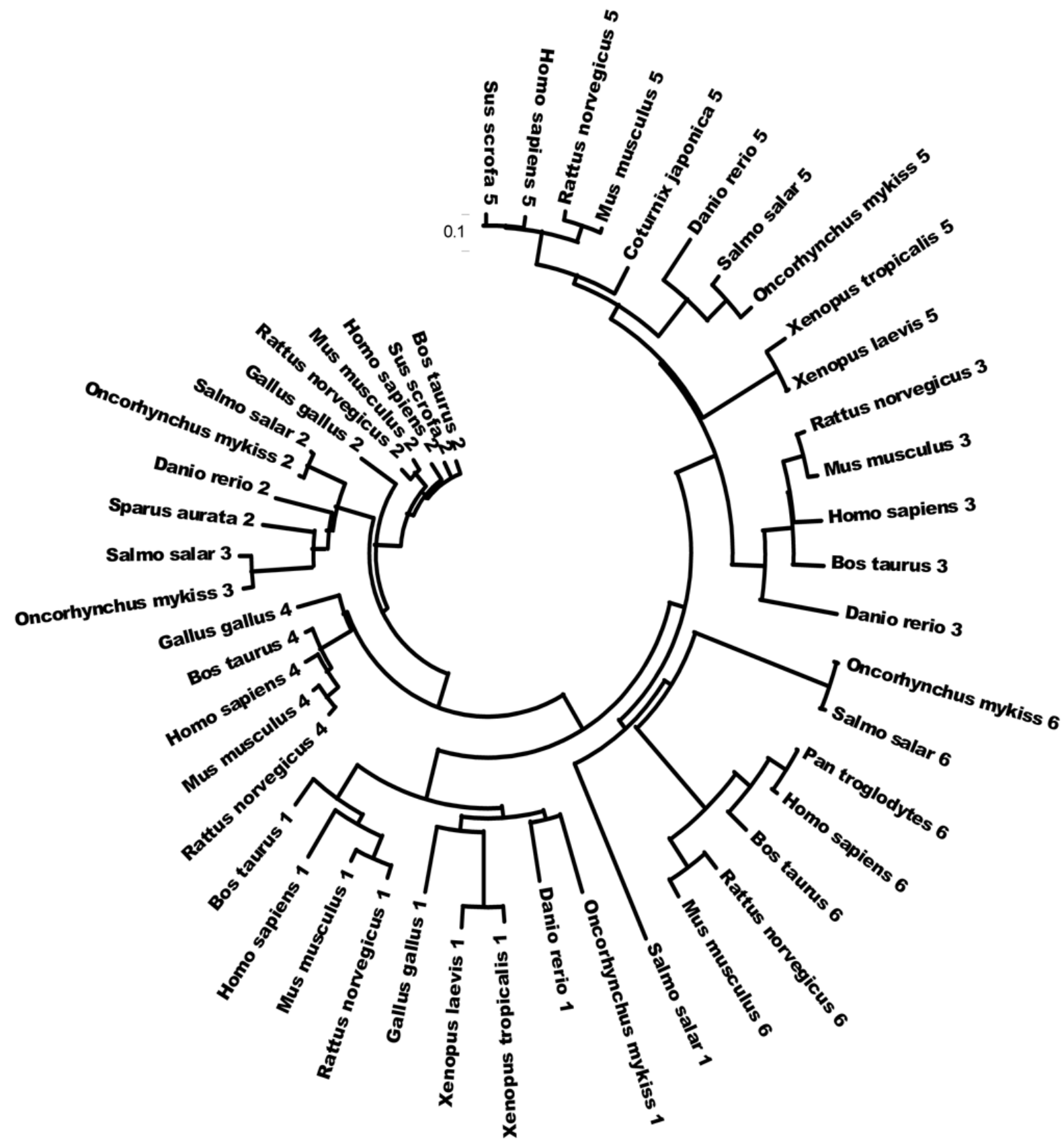

Figure 1.

Phylogenetic analysis of IGFBP sequences from twelve different species. This unrooted circular tree demonstrates IGFBP-2 as the central most subfamily (and thus the closest member to the IGFBP progenitor), followed by IGFBP-4 as the next closest relative. IGFBPs 1, 3, and -5 are near equidistant from IGFBP-4. While there were no full length IGFBP-4 sequences available from a fish species for this analysis, we note the rainbow trout IGFBP-4 has been partially cloned (consistent with the theory that all six IGFBPs originated in aquatic vertebrates). 


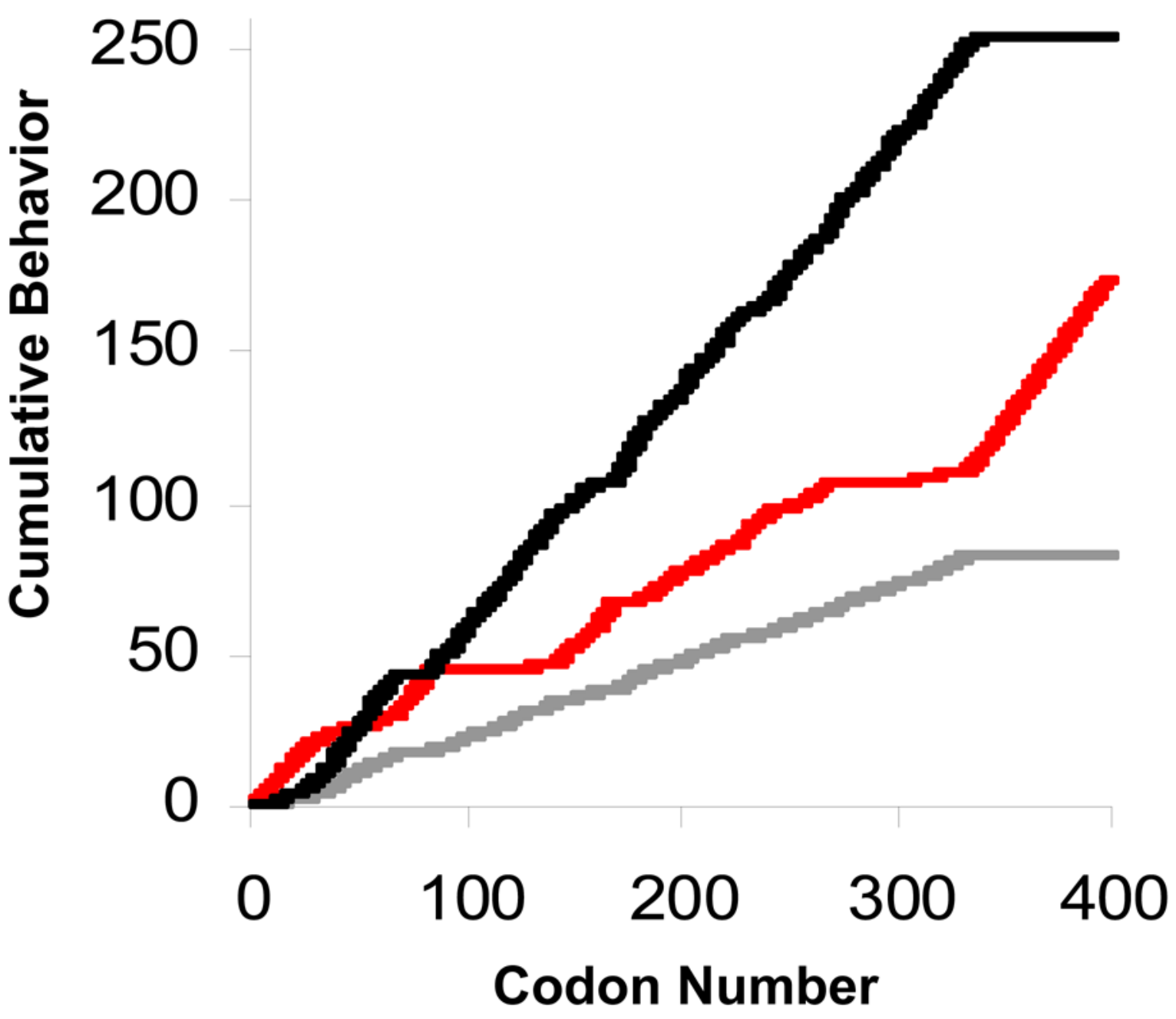

Figure 2.

Cumulative mutational behavior by codon in the IGFBP gene family. The black line represents non-synonymous cumulative mutations. The red line represents cumulative insertions and deletions. The gray line represents synonymous cumulative mutations. Non-synonymous mutations occur commonly throughout most of the gene. Insertions and deletions occur more episodically and synonymous mutations occur with the least frequency. These findings indicate that IGFBPs have experienced intense selection pressure for divergence. 


\section{Cumulative behavior}

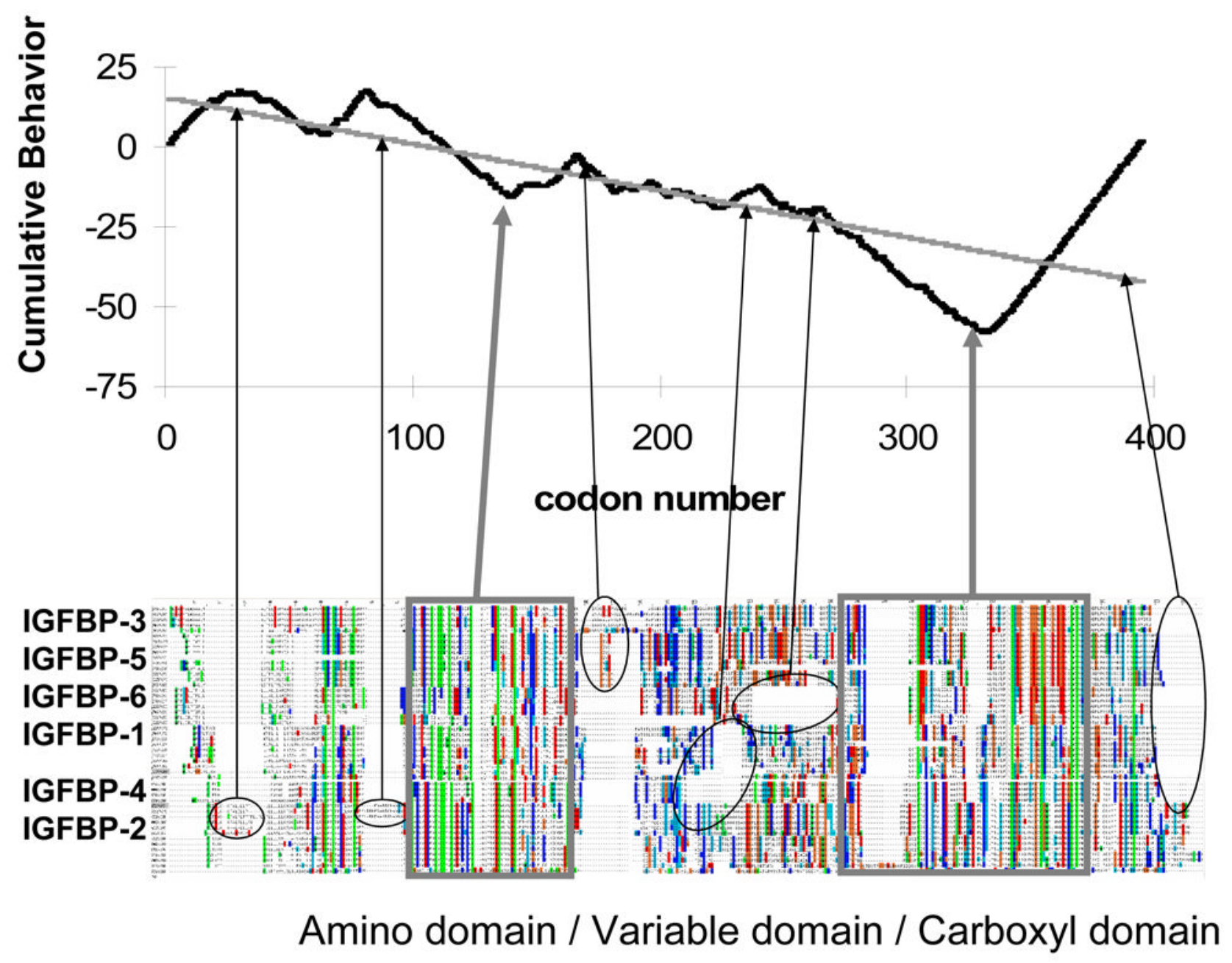

Figure 3.

Summary integral of cumulative non-synonymous mutations plus cumulative insertion / deletion mutations, minus synonymous mutations (black line). The plot includes a linear regression (gray line) that bisects the function, better defining peaks of selection pressure versus valleys of conservation. These peaks and valleys have been manually matched to a composite display of the multisequence alignment of all 78 full length IGFBP amino acid sequences (which display negatively charged amino acids in red/orange, positively charged amino acids in dark blue/light blue and sulfur containing amino acids in green/ochre). Black ovals represent insertions or deletions that correspond with peaks of selection pressure. Gray boxes represent cysteine rich regions that correspond with valleys of conservation. Insertion / deletion mutations are present within a variable domain hot spot for every IGFBP subfamily except IGFBP-2 (ovals), suggesting that these insertion / deletion events may have been important for divergence of subsequent IGFBP subfamilies. 